\title{
Determining the Effect of Smartphone Alerts and Warnings on Street-Crossing Behavior in Non-Mobility-Impaired Older and Younger Adults
}

\author{
Jeehan Malik \\ Computer Science, University of Iowa
}

\author{
Hanxi Tang \\ Psychological and Brain Sciences, \\ University Of Iowa
}

\author{
Morgan N. Di Napoli Parr \\ Psychological and Brain Sciences, \\ University Of Iowa
}

Joseph K. Kearney

Computer Science, University of Iowa

Kyle Rector

Computer Science, University of Iowa

\author{
Jessica Flathau \\ Psychological and Brain Sciences, \\ University Of Iowa \\ Jodie M. Plumert \\ Psychological and Brain Sciences, \\ University Of Iowa
}

\begin{abstract}
Vehicle manufacturers and government agencies are considering using vehicle-to-pedestrian (V2P) communication to improve pedestrian safety. However, there are unanswered questions about whether people will heed alerts and warnings presented through a smartphone. We conducted between-subject studies with younger and older adults where they physically crossed a virtual street. They received either permissive alerts (safe to cross), prohibitive warnings (not safe to cross), or no alerts or warnings (control). We found that both older and younger adults were highly likely to heed permissive alerts, even when this meant taking gaps between two vehicles that were smaller than they would typically take on their own. We also found that we could shift participants' road-crossing behavior toward greater caution when they were only alerted to cross very large gaps between two vehicles. Participants stated that alerts and warnings were useful, but that prohibitive warnings were annoying. These findings give insights into V2P design and pedestrian behavior when smartphone assistance is provided.
\end{abstract}

\section{CCS CONCEPTS}

- Human-centered computing $\rightarrow$ Human computer interaction (HCI); Empirical studies in $\mathrm{HCI}$; Social and professional topics $\rightarrow$ User characteristics; Age; Seniors.

\section{KEYWORDS}

Older Adults, Younger Adults, Smartphone Alerts, Pedestrian Street Crossing, Virtual Environments

\footnotetext{
Permission to make digital or hard copies of all or part of this work for personal or classroom use is granted without fee provided that copies are not made or distributed for profit or commercial advantage and that copies bear this notice and the full citation on the first page. Copyrights for components of this work owned by others than ACM must be honored. Abstracting with credit is permitted. To copy otherwise, or republish, to post on servers or to redistribute to lists, requires prior specific permission and/or a fee. Request permissions from permissions@acm.org.

CHI '21, May 08-13, 2021, Yokohama, Japan

(C) 2021 Association for Computing Machinery.

ACM ISBN 978-1-4503-8096-6/21/05 . .\$15.00

https://doi.org/10.1145/3411764.3445234
}

ACM Reference Format:

Jeehan Malik, Morgan N. Di Napoli Parr, Jessica Flathau, Hanxi Tang, Joseph K. Kearney, Jodie M. Plumert, and Kyle Kasie Rector. 2021. Determining the Effect of Smartphone Alerts and Warnings on Street-Crossing Behavior in Non-Mobility-Impaired Older and Younger Adults. In CHI Conference on Human Factors in Computing Systems (CHI '21), May 08-13, 2021, Yokohama, Japan. ACM, New York, NY, USA, 12 pages. https://doi.org/10.1145/3411764. 3445234

\section{INTRODUCTION}

Pedestrian injuries and deaths caused by collisions with motor vehicles are a major concern worldwide, with the number of annual road traffic deaths reaching 1.35 million [44]. In the U.S., the number of pedestrians killed along roadways in 2018 was the highest since 1990 [22]. Most fatalities occur in urban areas where the risk of potential conflicts between pedestrians and vehicles is high. Fatal collisions are more likely to occur at mid-block locations than at controlled intersections [37]. Further, $21 \%$ of all pedestrian fatalities in 2018 were people 65 years and older [26]. Older adults are at increased risk of traffic deaths due to greater risk for collisions and poorer recovery outcomes [30].

Governmental agencies and car manufacturers are testing sophisticated vehicle technology around the world [6, 18, 42, 43]. For instance, Vehicle-to-Vehicle (V2V) communication technology has completed the first phase of its pilot in New York, where 8,000 vehicles were equipped [29]. With advanced sensing technology, alert and warning systems are already being developed for drivers [39], with just a matter of time before such systems are developed for vulnerable road users such as pedestrians and cyclists [41]. There is growing interest in how pedestrians use traffic information communicated to a handheld device from either vehicles or roadway infrastructure [41]. Given the ubiquity of personal handheld devices and the limited numbers of vehicles equipped with eHMI (electronic human-machine interfaces), it is important to understand how pedestrians might respond to traffic information delivered via a handheld device.

Recent HCI research has explored how pedestrians interpret and respond to eHMIs containing cues in different modalities on or around Automated Vehicles (AVs) to convey their awareness 
and intent $[1,14,23]$. V2P communication also allows vehicles to communicate their location to a pedestrian's smartphone via Dedicated Short-Range Communications technology or other wireless technologies $[2,17,20]$. This information can then be used to generate an alert or warning for the pedestrian. Research has shown that providing smartphone warnings and alerts to younger texting pedestrians about whether a gap between two vehicles is safe to cross can improve road-crossing behavior in a virtual environment [35]. V2P communication can inform older adults (ages 65+) as well, given that older adult smartphone usage increased by $24 \%$ between 2013 and 2017 [4]. However, little is known about how older adults respond to smartphone alerts and warnings when making road-crossing decisions.

We examined the effect of smartphone alerts and warnings on the behavior of younger (defined as college-age) and older (defined as $65+$ years of age) pedestrians while crossing a stream of continuous traffic at a mid-block location. As a first step in this area of research, we worked with both older and younger adults who do not use mobility aids when walking. For our study, we used a large-screen immersive virtual environment commonly known as a CAVE [9]. Using a between-subjects design, we asked younger and older adults to cross while receiving either permissive alerts (informing when it was safe to cross), prohibitive warnings (informing when it was not safe to cross), or no alerts or warnings (as a control). These alerts and warnings were ability based, and the thresholds for the alerts and warnings were based on individuals' brisk walking speed, assessed prior to the road-crossing task. We discovered that both younger and older participants heeded the permissive alerts, but less so the warnings. Further, while participants with alerts safely crossed gaps between two vehicles (referred to as "gaps") with a substantial time to spare, they took smaller gaps than participants who received warnings or were in the control condition. In response, we added a between-subjects condition to determine whether we could influence participants to take only very large gaps using non-ability-based permissive alerts (called the "cautious permissive alerts" condition). Both older and younger adults took significantly larger gaps between two vehicles when they received cautious permissive alerts than when they received the original permissive alerts, even with lower numbers of participants due to halting the study for the COVID-19 pandemic.

Our empirical findings show that both younger and older adults are highly compliant in their responses to permissive alerts communicated via a smartphone. We close by discussing age-related similarities and differences and how our results inform the design of V2P systems, particularly the timing of alerts and warnings communicated to pedestrians when crossing continuous streams of traffic.

\section{BACKGROUND AND RELATED WORK}

\subsection{Road Crossing Characteristics for Younger and Older Adults}

Previous research has explored the road-crossing decisions of both younger and older adults either by having participants step/walk in a virtual setting $[12,45]$ or by pressing a computer key while watching a video of traffic [32]. This research shows that older adults both choose unsafe gaps and miss crossing opportunities. Zito et al.
[45] found these unsafe crossings and missed opportunities were for both fast and slow cars, while Dommes et al. [12] found that the older adult groups made more unsafe decisions than the younger adults with high car speeds $(50 \mathrm{~km} / \mathrm{h}+)$, and older adults between 61 and 71 years missed more opportunities to cross with slower car speeds $(30 \mathrm{~km} / \mathrm{h})$. Further, Naveteur et al. [28] showed that older adults take longer when stepping from a curb to cross the street and that this delay increases with age and fear of falling. This research indicates that older adults have more difficulty than younger pedestrians in making road-crossing decisions and underscores the need to learn about the effect of V2P technologies on both younger and older pedestrians, especially given that both road infrastructure (including instrumented roadways) and vehicles are rapidly changing due to technological advances [41].

\subsection{Communicating Vehicle Intent and Awareness with External Human-Machine Interfaces (eHMI)}

Studies have examined optimal designs for eHMI communication and pedestrian responses to eHMI designs. Mahadevan et al. took a pedestrian-centered approach to explore how to communicate the intent of oncoming vehicles to pedestrians at a crosswalk [23]. They proposed a design space for autonomous vehicle-pedestrian interactions including having visual (e.g., text, color), auditory (e.g., human-like voices or sounds on the pedestrian's phone), and physical (e.g., haptic cues) modalities. They found that participants preferred to receive explicit information about a vehicle's awareness and intent instead of solely relying on the vehicle's movement (i.e., slowing down), while there was a split opinion about whether they would like to receive information through the vehicle, or a phone held in their hand. Faas et al. [14] conducted a longitudinal video study of the effects of eHMIs and similarly found that pedestrians benefit from eHMIs that communicate intent and status, and felt safer with the eHMIs. Ackermans et al. [1] compared the effect of a conspicuous sensor system on the roof of a vehicle and an eHMI in front of a vehicle on pedestrian attitudes and willingness to cross in a VR environment. They found that eHMIs improved participants' willingness to cross when a vehicle was yielding and improved participants' recognition of non-yielding vehicles when a vehicle did not intend to yield. eHMIs have the potential to help pedestrians, but pedestrians must be within eyesight or hearing distance of the vehicle to benefit from eHMI information.

\subsection{Vehicle-to-Pedestrian (V2P) Communication Using Smartphones}

V2P technology can improve traffic safety by alerting pedestrians about potential collisions. In fact, the U.S. National Highway Transportation Safety Administration proposed to mandate that all new vehicles require such technology [27]. They estimate V2P communication for both drivers and pedestrians could reduce pedestrian crashes by up to $46 \%$ [41]. This technology can be particularly useful when driver or eHMI cues are not available or are imperceptible.

V2P communication has the potential to inform older adults about when it is safe or risky to cross a roadway. Over $75 \%$ of smartphone owners above 50 years of age say that they use smartphones to get directions or traffic information [3], suggesting that they 
may be receptive to using smartphone apps to help them make street-crossing decisions. In a study of older adults, Felberbaum et al. [16] conducted semi-structured interviews and focus group sessions with adults aged 73-90 to uncover design requirements for V2P communication. They found that the technology should run automatically without requiring the user to learn it, encourage mobility of the user within the environment in which they are using the device, and be unobtrusive enough such that a user can access the technology and walk simultaneously. We employ these V2P communications design considerations and in turn provide empirical evidence of how these designs impact the street-crossing behaviors for younger and older adults.

\subsection{Effect of Smartphone Alerts and Warnings on Street-Crossing Behavior of Texting Younger Adults}

At present, little is known about how people respond to smartphone alerts and warnings for guiding street-crossing behavior. One exception is Rahimian et al. [35, 36] which examined whether V2P communication via a smartphone could mitigate the harmful effects of texting on pedestrian road-crossing safety. They conducted two experiments in which college-age participants physically crossed a virtual roadway while texting on a smartphone. The first study informed texting participants with a permissive alert when a safe gap was approaching by using a countdown clock and an auditory alert on the phone. Participants who received alerts selected larger gaps and had more time to spare when they crossed the road than texting participants who did not receive alerts. However, texting participants who received alerts spent much less time looking at oncoming traffic, possibly indicating an overreliance on the technology. The second study informed texting participants with a prohibitive warning when they began to cross a dangerously small gap by playing an auditory warning on the phone. Participants in the warning group waited significantly longer to cross and chose larger gaps for crossing compared to the no texting and texting without warnings groups, However, participants who received a warning after they entered the road never reversed their motion and returned to the side of the road.

These two studies provide insight into how smartphone alert and warning systems can positively affect road-crossing behavior. However, these studies were only based on a younger population (college-age students), and the smartphone alerts and warnings used a one-size-fits-all approach in terms of the size of gaps alerted or warned. With older adults this approach may not work because older adults may walk at different speeds [40]. Here, we examine how ability-based alerts and warnings (based on individual walking speeds) delivered via a smartphone influence older and younger pedestrians' street-crossing behavior in a virtual environment.

\section{ALERT AND WARNING DESIGN AND IMPLEMENTATION}

We generated smartphone alerts and warnings based on a participant's pre-recorded brisk walking pace in a nearby hallway at the beginning of the test. We used this pace to estimate the time it would take them to cross the 10 -foot $(3.048 \mathrm{~m})$ "road" in our virtual environment (known as a CAVE [9]; see Section 4.1). We created a time buffer of 1 second to account for the older adults' slower timing of entry based on research showing that the timing of entry (or how tightly a person enters the gap relative to the lead car) was 0.65 seconds for younger adults and 0.9 seconds for children [31]. We also added a 1-second buffer as a safe time to spare relative to the tail car when exiting the roadway. This resulted in 2-second total buffer added to the individualized crossing time estimate for participants.

To implement the smartphone alerts and warnings, we developed an Android application in C\# (installed on a Huawei smartphone running on the EMUI 4.0 operating system). The smartphone communicated with the system through a wireless network using TCP/IP. When the smartphone did not display an alert, the screen was black.

\subsection{Permissive Alerts}

The system determines it is safe to cross when a person's crossing time estimate is less than the time before the tail car of the next gap drives through the crosswalk of our virtual environment. We define 'lead car' as the car that passed just before the participant crossed, and 'tail car' as the oncoming/next car. The system sends a message to the smartphone to display a permissive alert consisting of a black screen with green text on it saying, "SAFE TO CROSS!" (Figure 1). The smartphone plays a 0.8 -second audio message saying, "Safe to cross" right before the safe gap opens, such that the audio message would finish as the safe gap was in front of the person. Alongside the display and audio, the phone vibrates three times for $0.5 \mathrm{~s}$ each. We chose repetitive 0.5 -second vibrations due to Arab et al. [5] reporting that $78 \%$ participants considered haptic repetitions useful to 'emphasize the imminence of an event' in an emergency situation. While permissive alerts are not an emergency, we chose to parallel our prohibitive warnings (described below). The visual display stays on until the gap is no longer safe to cross.

\subsection{Prohibitive Warnings}

The system determines it is not safe to cross when a person's crossing time estimate is greater than the time before the tail car of the next gap drives through the crosswalk in our virtual environment. The system sends a message to the smartphone to display a prohibitive warning consisting of a red screen with white text on it saying, "DO NOT CROSS!" (Figure 1). The smartphone plays a 0.8 -second audio message saying, "Do not cross" right before the safe gap closes, such that the audio message would finish as the safe gap closed in front of the person. Alongside the display and audio, the phone vibrates three times lasting $0.5 \mathrm{~s}$ each. The visual display stays on and the audio message repeats every 2 seconds until it is safe for the person to cross again. The warning duration could include more than one gap when there are a series of uncrossable gaps.

\section{VIRTUAL ENVIRONMENT AND TASK SCENARIO}

\subsection{Physical Setting}

We conducted the experiment in a large-screen virtual environment with three screens placed at right angles to each other, forming a 


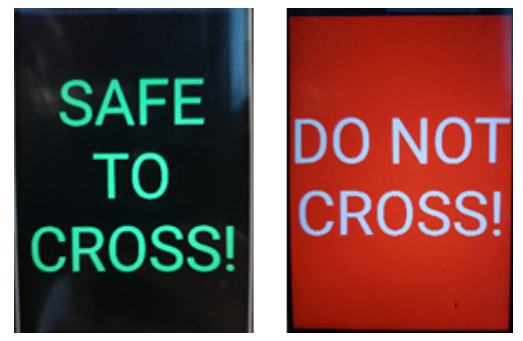

Figure 1: Permissive alert visual display (left) and prohibitive warning visual display (right).
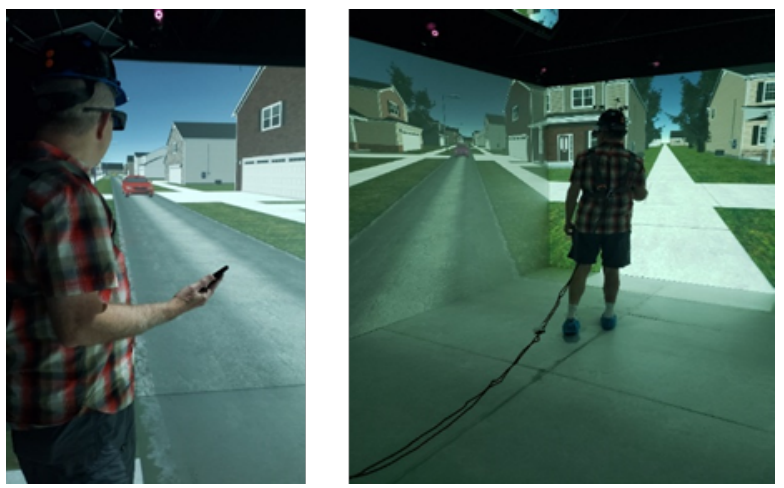

Figure 2: A person using the smartphone alert system in our street-crossing scenario. Note that the visual angles are correct from the viewpoint of the user.

three-walled room (4.33m long x $3.06 \mathrm{~m}$ wide $\times 2.44 \mathrm{~m}$ high). The length of the side screens allowed participants to physically walk across a 10 -foot-wide $(3.048 \mathrm{~m})$ virtual road. Three DPI MVision 400 Cine 3D projectors back-projected high-resolution (1920x1080) graphics in stereo onto the screens. An identical projector frontprojected high-resolution (1920x1080) stereo images onto the floor $(4.33 \times 3.06 \mathrm{~m})$.

Participants wore stereo shutter glasses, synchronized with the displays. We used an OptiTrack Flex13 motion capture system to determine the position and orientation of the participant's head based on reflective markers mounted on a helmet worn by the participant (Figure 2). This system allowed us to render the images with the correct perspective for participants as they moved. Participants also wore a harness connected to a post at the back of the simulator to ensure that they would not run into the front screen (Figure 2). To play car sounds, we used Logitech THX certified speakers as a surround sound system. The speakers generated spatialized traffic sounds.

\subsection{Virtual Traffic Scenario}

The virtual traffic scenario was a one-lane road in a residential neighborhood. Participants stood at the edge of the roadway and viewed continuous $25 \mathrm{mph}(11.176 \mathrm{~m} / \mathrm{s})$ traffic traveling from left to right (Figure 2). The task was to physically cross the virtual roadway without colliding with a vehicle. We chose this car speed based on previous work indicating that older pedestrians underestimate their ability to cross at car speeds lower than $30 \mathrm{~km} / \mathrm{h}(\sim 18.64 \mathrm{mph})$ and overestimate their ability to cross at speeds higher than $50 \mathrm{~km} / \mathrm{h}$ ( 31.07 mph) [12]. The randomly ordered temporal gaps between cars ranged in size from 2 to 7 seconds (similar to that used by Dommes et al. [13]). We built the traffic scenario using the Unity3D gaming platform and recorded the positions and orientations of vehicles and the participants.

\section{COMPARING ABILITY-BASED ALERTS AND WARNINGS WITH CONTROL}

We conducted a between-subjects experiment where the independent variable was the mobile alert type with three conditions: 1) Permissive alerts: Participants received alerts telling them when it was safe to cross; 2) Prohibitive warnings: Participants received warnings telling them that it was not safe to cross; and 3) Control: Participants did not receive any alerts or warnings. In all conditions, participants carried a smartphone in their hand. We chose a between-subjects design to control for possible carry-over effects of experience with alerts and warnings. In addition, because our aim was to study the effects of multimodal alerts/warnings alone on older and younger pedestrians' street-crossing decisions, we did not give participants extra phone tasks.

\subsection{Participants}

We recruited 72 younger adults and 71 older adults. The younger adults were recruited from a psychology course at a university and received course credit for participating. The older adults were recruited using mass emails sent to a university community, including both current and retired employees. Our inclusion criteria for older adult participants were that their age was $65-85$, they were fluent in written and spoken English, had normal or corrected-to-normal vision and hearing, and could walk unassisted. We also screened older adults for dementia over the phone using a brief version of the Mini Mental State Exam (MMSE) [33]. We only included older adults as participants if they scored at least 14 out of 16 correct (of which 7 older adults did not qualify).

We randomly assigned each participant to a condition, with 24 participants in each condition: permissive, prohibitive, and control, with the exception that the control condition had 23 older adults. A Kruskal-Wallis test comparing the older adults in the three conditions in terms of gender, age, smartphone experience, and walking experience revealed no significant differences. The same was true when comparing younger adults in the three conditions. We present the demographic information in Table 1

\subsection{Procedure}

After providing informed consent, the experimenter asked participants to walk briskly down a hallway between two marked lines while timing with a stopwatch. Participants performed the baseline walking task twice to ensure a stable estimate of brisk walking speed. The average baseline brisk walking speed and walking speeds in the virtual environment were significantly correlated $(\mathrm{r}=.49, \mathrm{p}<.001)$.

After the baseline walking task, participants performed the virtual road-crossing task. We first fitted them with the tracking helmet, shutter glasses, and harness. Each trial began without any 
Table 1: Participant age, gender, smartphone ownership, and average smartphone comfort level and skill level for each condition. Comfort was reported from $1=$ not at all comfortable to $5=$ very comfortable. Skill was reported from $1=$ not at all skillful to 5 = very skillful.

\begin{tabular}{lllllll}
\hline Condition & Age Group & Age Range (Mean) & M/F & $\begin{array}{l}\text { Own } \\
\text { Smartphone }\end{array}$ & $\begin{array}{l}\text { Smartphone } \\
\text { Comfort }\end{array}$ & $\begin{array}{l}\text { Smartphone } \\
\text { Skill }\end{array}$ \\
\hline Permissive & Younger Adults & $18-21(18.5)$ & $12 / 12$ & $100 \%$ & $4.88(0.33)$ & $4.50(0.50)$ \\
Prohibitive & Younger Adults & $18-24(18.8)$ & $12 / 12$ & $100 \%$ & $4.73(0.43)$ & $4.29(0.56)$ \\
Control & Younger Adults & $18-22(18.9)$ & $12 / 12$ & $100 \%$ & $4.88(0.36)$ & $4.31(0.66)$ \\
Permissive & Older Adults & $65-85(71.8)$ & $11 / 13$ & $96 \%$ & $4.04(0.85)$ & $3.59(1.14)$ \\
Prohibitive & Older Adults & $65-84(69.2)$ & $12 / 12$ & $92 \%$ & $4.25(0.82)$ & $3.60(0.77)$ \\
Control & Older Adults & $65-83(71.7)$ & $9 / 14$ & $83 \%$ & $3.89(1.01)$ & $3.68(0.99)$ \\
\hline
\end{tabular}

traffic on the road. Participants stood on the edge of the roadway and viewed a continuous stream of vehicles approaching from the left. Participants were told that they should wait for the first car to pass (to prevent them from crossing in front of the stream of traffic) but then they could wait as long as they wanted before attempting to cross the road without being "hit" by a vehicle. Once they selected a gap to cross, participants physically walked to the other side of the road. Traffic generation ceased once they reached the sidewalk on the other side of the road, allowing them to return to the starting position.

The road-crossing task began with three practice trials in which the experimenter walked with participants. On the first practice trial, participants crossed without traffic or a smartphone to become familiar with the virtual environment. On the second trial, participants crossed with traffic but without a smartphone. On the third trial, participants crossed with a smartphone in their hand. The smartphone was inactive for the Control group. For the Permissive and Prohibitive groups, we turned on the smartphone and gave a demo of the alert to familiarize participants with the sight, sound, and feel of the alert or warning. Participants in the permissive condition were instructed that the smartphone would give them an alert when it was safe to cross and participants in the prohibitive condition were instructed that the smartphone would give them a warning when it was not safe to cross.

After the practice trials, participants completed 20 experimental trials in which they crossed on their own. The road-crossing task took about 20 minutes to complete. After finishing the road-crossing task, we conducted a 10-minute interview about the participants' experience with the alerts/warnings. We asked questions about what modality (visual, auditory, vibratory) they noticed first and what was most prominent. We also asked about likes, dislikes, and improvements about the alerts/warnings. Lastly, participants filled out a survey that we proctored on Qualtrics [34] that asked them about their pedestrian habits and smartphone experience (addressed in Section 5.1). After completing the survey, we debriefed them about the study.

\subsection{Data Recording and Measures}

Every $\sim 0.02 \mathrm{~s}$ (or a $\sim 50 \mathrm{~Hz}$ sampling rate), we recorded the position of the participant's head and all vehicles, and the times at which the system sent messages to the phone. In addition to recording which gap was taken, we also measured: 1) Timing of entry: time between the participant and the rear of the lead car in the gap when the participant entered the swath of the cars (i.e., how tightly participants timed their entry into the gap relative to the lead car). 2) Road crossing time: time between when the participant entered and exited the swath of the cars. 3) Time to spare: time between the participant and the front of the tail car in the gap when the participant cleared the swath of the cars (or the margin of safety when exiting the roadway). We recorded a collision when time to spare was $\leq 0$ seconds but did not analyze them because only one happened during a permissive trial $(0.032 \%$ of all trials).

We determined whether participants complied with the alerts and warnings (or lack thereof). We measured non-compliance in two ways. First, we calculated "errors of omission" where participants chose not to cross the road when the alert was on or the warning was off. These are missed opportunities to cross. These errors are less important because a participant is choosing to be cautious. Second, we calculated "errors of commission" where participants ignored the alerts or warnings. This happened when participants crossed when the alert was off or when the warning was on. These errors are important because they may have inadvertent consequences in real-world road-crossing situations.

\subsection{Data Analysis}

Our primary goals were to determine whether there were significant differences between the permissive and prohibitive conditions as compared to the control condition and whether performance was related to age. We used mixed-effects logistic regression analyses to model the likelihood of accepting (or rejecting) each gap that was seen on all 20 trials as a function of condition, age, and gap size. Assumptions of linearity, normality of residuals, and homogeneity of variance were met for our mixed-effects models. The final model included fixed-effects predictors of condition and age (as a categorical variables) and gap size (as a continuous variable), and a random intercept for participant and a random slope for gap size. We examined the continuous measures of movement timing using separate mixed-effects linear regression analyses to model the effects of condition and age on timing of entry, crossing time, and time to spare (across the 20 trials). The final model included fixedeffects predictors of condition and age (as categorical variables) and a random intercept for participant. The intraclass correlation coefficients (ICC) were .26 for timing of entry, .80 for crossing time, and .21 for time to spare. 

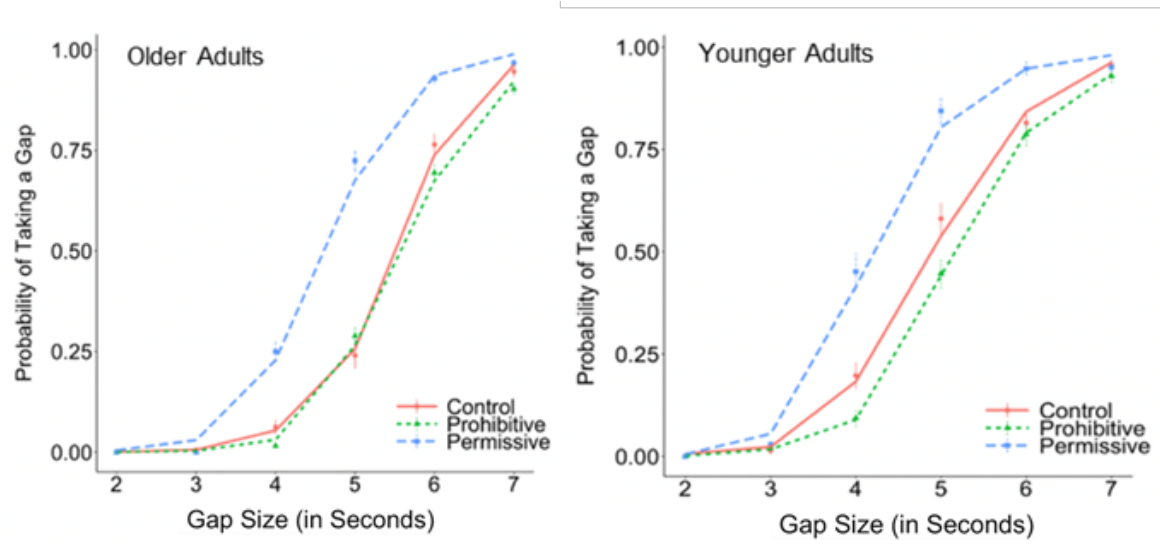

Figure 3: Logistic regression curves modeling the probability of taking a gap as a function of condition and gap size for older adults (left) and younger adults (right).

We audio-recorded and transcribed the interviews with categorical responses and open-ended responses. Because the categorical data were non-normally distributed, we conducted Mann-Whitney U-tests between the alert and warning groups. We conducted open coding [7] of the transcripts. Two researchers independently read the transcripts and identified themes. The researchers then synthesized a set of codes including aspects liked and disliked and suggestions for improvement. The researchers coded each interview response individually. The researchers met regularly to discuss each other's codes until they agreed on all code assignments and revised the codes, as necessary. Due to both researchers discussing all codes together, we did not calculate inter-rater reliability.

\section{QUANTITATIVE RESULTS}

\subsection{Road-Crossing Measures}

Figure 3 shows the results of mixed-effects logistic regression analyses modeling the likelihood of taking a gap. There was an overall effect of age group $(b=1.59, z=2.59, p<.01)$ such that older adults selected larger gaps to cross than younger adults. An effect of gap size indicated that participants preferred larger over smaller gaps $(z$ $=10.97, p<.001$ ), with 21.32 increased odds of accepting a gap with each 1-second increase in gap size. We also found that participants in the control condition selected larger gaps than participants in the permissive condition $(b=2.42, z=3.92, p<.001)$, but not in the prohibitive condition ( $b=-.47, z=-.76, \mathrm{~ns})$. This pattern held across both age groups. Older and younger adults in the permissive condition $(b=2.35, z=5.17, p<.001$, and $b=1.87, z=2.92, p<.01$, respectively) selected smaller gaps than their counterparts in the control condition.

We used linear mixed-effects regression to model the effects of age group and condition on timing of entry, road crossing time, and time to spare. Participants in the permissive condition had significantly less time to spare than those in the control condition $(t(138.50)=-2.97 p=.003)$, which was expected based on the differences in gap choices seen above. Condition had no effect on timing of entry or road crossing time, and age was not a predictor of any movement timing variables. Table 2 has descriptive statistics
Table 2: Means and standard deviations (in parentheses) of movement timing measures (in seconds) for each condition.

\begin{tabular}{lccc}
\hline Condition & Timing of Entry & Crossing Time & Time to Spare \\
\hline Control & $.77(.23)$ & $2.11(.26)$ & $3.34(.83)$ \\
Prohibitive & $.87(.30)$ & $2.12(.25)$ & $3.32(.85)$ \\
Permissive & $.72(.21)$ & $2.14(.26)$ & $3.05(.95)$ \\
\hline
\end{tabular}

for the timing variables averaged across younger and older adult participants.

\subsection{Alert Compliance}

For permissive alerts, we found that both younger and older participants were highly compliant (see Table 3 for raw count). When they chose to cross the road, both older and younger adults virtually always did so when an alert was on, resulting in very small errors of commission: only $0.41 \%$ for older adults and only $1.6 \%$ for younger adults. (Note that the reason older adults selected larger gaps than younger adults, and yet both groups were "highly compliant" is because the alerts were ability-based: $75 \%$ of younger adults but just $54 \%$ of older adults were alerted to gaps of $4+$ seconds. Thus, we would expect to see an age difference in gap choice.) When they chose not to cross the road, both younger and older adults mostly did not do so when the alert was off, but they also sometimes failed to cross when the alert was on. These errors of omission (i.e., "missed opportunities") were $22.6 \%$ for older adults and $21.5 \%$ for younger adults.

Similarly, for prohibitive alerts, we found that both younger and older participants were highly compliant (see Table 4 for raw count). When they chose to cross the road, both older and younger adults nearly always did so when the warning was off. Notably, like the permissive condition, we found very small errors of commission at $0.21 \%$ for older adults and $1.9 \%$ for younger adults. When they chose not to cross the road, both younger and older adults had a substantial number of missed opportunities (not crossing when the 
Table 3: The number of trials that older adults and younger adults complied or did not comply with the permissive alerts.

\begin{tabular}{lcccc}
\hline Alert Status & Older: did not cross & Older: crossed & Younger: did not cross & Younger: crossed \\
\hline Alert Off & 332 & 2 & 284 & 8 \\
Alert On & 97 & 478 & 78 & 473 \\
\hline
\end{tabular}

Table 4: The number of trials that older adults and younger adults complied or did not comply with the prohibitive warnings.

\begin{tabular}{lcccc}
\hline Warning Status & Older: did not cross & Older: crossed & Younger: did not cross & Younger: crossed \\
\hline Warning Off & 233 & 471 & 360 & 472 \\
Warning On & 423 & 9 & 459 & 1 \\
\hline
\end{tabular}

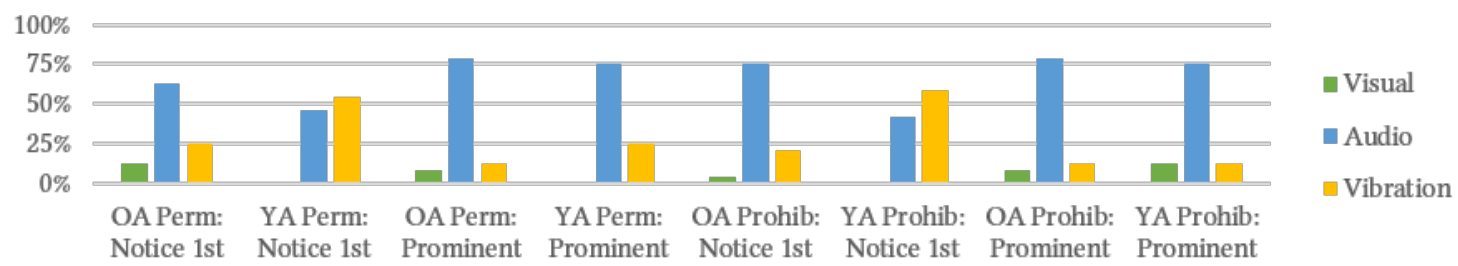

Figure 4: Percentage of older adult (OA) and younger adult (YA) participants who reported whether they noticed visual (left, green), audio (middle, blue), or vibration (right, yellow) first or thought they were most prominent for the permissive alerts and prohibitive warnings. One older adult did not respond to prominence for permissive alerts.

warning was off). We found the errors of omission were $44.0 \%$ for older adults and $35.5 \%$ for younger adults.

\section{QUALITATIVE RESULTS}

\subsection{Categorical Responses}

Participants reported which elements of the alerts/warnings (visual, auditory, or vibratory) they noticed first and were most prominent. Figure 4 shows the percentages. There were no statistically significant differences between the conditions when assessing each age group.

\subsection{Open-Ended Responses}

We found that older adult participants had more positive statements about the permissive alerts $(n=72)$ than the prohibitive warnings $(n=59)$ and had fewer negative comments about the permissive alerts $(n=41)$ than the prohibitive warnings $(n=51)$. Younger adult participants were more balanced, with both positive comments for alerts $(n=69)$ and warnings $(n=73)$ and negative comments for alerts $(n=38)$ and warnings $(n=47)$. Below, we describe the perceived benefits of alerts and warnings followed by their perceived risks and drawbacks. We will refer to older and younger adult participants in the permissive group as OPe1-OPe24 and YPe1-YPe24. We will refer to older and younger adult participants in the prohibitive group as OPr1-OPr24, YPr1-YPr24.

7.2.1 Benefits of the Permissive Alerts and Prohibitive Warnings. $16 / 24$ of older adult participants and 17/24 of younger adult participants mentioned that the permissive alerts were easy to understand and therefore helped them decide when to cross: "it was easy, it was like you just didn't have to think about it" [OPe3]. Fewer older adult participants felt the same way about the prohibitive alerts $(\mathrm{n}=11)$, while it was more balanced for younger adults $(\mathrm{n}=18)$ : " $I$ think they're good cause they help us to know when to cross and when not to" [YPr11].

The other recurring positive theme in both conditions was a match between participants' expectations of alert/warning behavior and performance. For permissive, 12/24 older adults and 10/24 younger adults made a positive comment. OPe1 reflected that "[The alerts] correlated with what I thought. .." YPe22 said, "most of the time I felt it was pretty accurate." Participants in the prohibitive condition also commented positively on trust, with $12 / 24$ older adults and 11/24 younger adults. For instance, OPr16 said the "[prohibitive warnings] certainly gave me enough time to cross."

7.2.2 Risks and Drawbacks of the Permissive Alerts and Prohibitive Warnings. Alarmingly, 5 older adults and 7 younger adults across both conditions mentioned that the alerts/warnings made them less attentive while crossing. OPe24 stated, “. . you kind of get passive and just wait for it to tell you what to do" and YPe14 states "...it was like I would wait for it to tell me when to walk." Another risk of the permissive alerts is that they sometimes encouraged older adults to cross when they were not as confident, which four older adults mentioned. OPe11 stated that the alerts "helped me cross but [I] wouldn't have gone sometimes if it didn't go off." Interestingly, YPr8 mentioned this phenomenon with the warnings: ". . .it just kind of gave me a little extra push to cross."

Even though participants complied with alerts and warnings, there were still negative comments about trust from older adults 
(10 in permissive, 12 in prohibitive) and younger adults (14 in permissive, 7 in prohibitive). For instance, OPe18 simply “. . . didn't trust them" [OPe18], while others had changing trust: "I didn't trust them at the beginning. And then after a while I just started to trust them more" [OPe16].

Almost half of the participants mentioned using their judgment over the alerts or warnings (23 older adults, 31 younger adults). There were no significant differences between the conditions regarding this sentiment for younger (13 in permissive, 18 in prohibitive) or older adults (11 in permissive, 12 in prohibitive). Older adult participants mentioned using the alerts or warnings in the future: "I think I would use them especially as I get older" [OPe6]. However, most participants spoke about relying on their own judgment solely or more than the alerts even though older and younger participants had small errors of commission in both the permissive and prohibitive conditions.

We also uncovered a notable difference between the permissive alerts and prohibitive warnings; 9/24 older adults mentioned that the prohibitive warnings were annoying, while no older adults mentioned this about the permissive alerts. OPr3 said: "once I got the rhythm and understood had it in my mind when it would be safe to cross and when it wouldn't then [the warnings] were annoying." Younger adults also thought the prohibitive warnings were annoying $(14 / 24)$ and less so for permissive alerts $(4 / 24)$, likely due to repetition: “. . After a while you're just like, ok, I'm tired of hearing that" [YPr13]. Younger adults thought permissive alerts were "really loud" [YPe16] and not appropriate to carry in public: "I probably wouldn't use the sound I'd just use the vibration because its more discreet." [YPe11].

\section{FOLLOW-UP CONDITION: CAUTIOUS PERMISSIVE ALERTS}

Our initial results indicated that both younger and older adult participants took smaller gaps in the permissive condition than in the control condition. Further, our qualitative findings uncovered a concern that participants may have crossed when they otherwise would not have with permissive alerts. Therefore, we conducted a follow-up between-subjects condition to test whether we could shift older and younger adults' gap acceptance thresholds in a more cautious direction by alerting them to only cross 6- or 7-second gaps (where crossing smaller gaps is considered 'non-compliant'). This condition was called "cautious permissive alerts," and followed the same protocols as the original permissive condition. Cautious permissive alerts were intentionally not ability-based because our goal was to determine whether we could influence their behavior to take only very large gaps, which all our previous participants were capable of crossing. We were particularly interested in comparing participant road-crossing performance in the new permissive condition with that in our original permissive condition.

\subsection{Participants}

We recruited 10 older adults and 20 younger participants using the same recruitment methods and inclusion criteria (see Section 5.1) before halting due to COVID-19 (see Table 5 for demographic information). We found a significant difference in the self-reported smartphone skill for younger adults between the four conditions
$(\mathrm{H}(3)=9.302, \mathrm{p}<.03)$. Bonferroni pairwise comparisons (alpha/6) indicated that younger participants self-reported a higher level of skill with a smartphone in the cautious permissive than in the original permissive condition $(\mathrm{W}=134.5, \mathrm{p}<.007)$. No other differences were significant.

\subsection{Quantitative Results}

8.2.1 Road-Crossing Measures (Permissive compared to Cautious Permissive). Figure 5 shows the results of mixed-effects logistic regression analyses modeling in the likelihood of taking a gap in the original permissive condition and the cautious permissive condition. There was a significant effect of condition $(b=-2.25 z=$ $-6.35, p<.001)$, indicating that participants in the original permissive condition took smaller gaps than those in the cautious permissive condition. An effect of gap size indicated that participants preferred larger over smaller gaps $(b=3.72, z=8.91, p<.001)$ with 41.38 increased odds of accepting a gap with each 1-second increase in gap size. Finally, there was a significant interaction between age, condition, and gap size $(b=2.18, z=.96, p=.02)$. For younger adult participants, there was a marginally significant Condition $x$ Gap Size interaction $(b=1.68, z=.92, p=.07)$. Relative to the original permissive condition, younger adults in the cautious permissive condition had 5.35 increased odds of accepting a gap with each 1-second increase in gap size. This indicates that younger adults in the cautious permissive condition were more discriminating in their gap choices than their counterparts in the original permissive condition (i.e., they had steeper gap acceptance slopes). There was also a marginal Condition $x$ Gap Size interaction for the older adults ( $b=-.99, z=-1.67, p=.09)$. Due to the small number of participants in older adult cautious permissive group, we did not analyze this result further. We found no effect of age group or condition on timing of entry, road crossing time, and time to spare. The intraclass correlation coefficients were .60 for timing of entry, .81 for crossing time, and .25 for time to spare. See descriptive statistics for the timing variables averaged across both younger and older adults in Table 6

8.2.2 Alert Compliance. For cautious permissive alerts, we found both younger and older adults were less compliant with the alerts compared to our original permissive condition (Table 7 has raw count). When they chose to cross the road, errors of commission (crossing when the alert was off) were at $8.1 \%$ for older adults and $17.8 \%$ for younger adults. Despite this rate being higher than participants in the original permissive condition (1.6\%), cautious permissive participants still selected larger gaps than the original permissive participants. This is because errors of commission in the cautious permissive condition are stricter than the original permissive condition (i.e., cautious permissive participants were not allowed to cross anything less than a 6-second gap, whereas previously they could cross smaller gaps based on their ability). In other words, the highly cautious permissive alerts could have had a side effect of being perceived as "too safe," making people choose to cross non-alerted (i.e., smaller) gaps. In contrast, we found that there were fewer "missed opportunities" than in the original permissive condition (older adults $=9.4 \%$ and younger adults $=2.2 \%$ ). This result makes sense because we alerted only very large gaps, so 
Table 5: Participant age, gender, smartphone ownership, and average smartphone comfort level and skill level for each condition. Comfort was reported from $1=$ not at all comfortable to $5=$ very comfortable. Skill was reported from $1=$ not at all skillful to 5 = very skillful.

\begin{tabular}{lllllll}
\hline Condition & Age Group & Age Range (Mean) & M/F & $\begin{array}{l}\text { Own } \\
\text { smartphone }\end{array}$ & $\begin{array}{l}\text { Smartphone } \\
\text { Comfort }\end{array}$ & $\begin{array}{l}\text { Smartphone } \\
\text { Skill }\end{array}$ \\
\hline Cautious Permissive & Younger Adults & $18-22(19.3)$ & $10 / 10$ & 20 & $4.88(0.31)$ & $4.76(0.40)$ \\
Cautious Permissive & Older Adults & $65-82(71.4)$ & $7 / 3$ & 10 & $4.55(0.65)$ & $3.80(0.60)$ \\
\hline
\end{tabular}
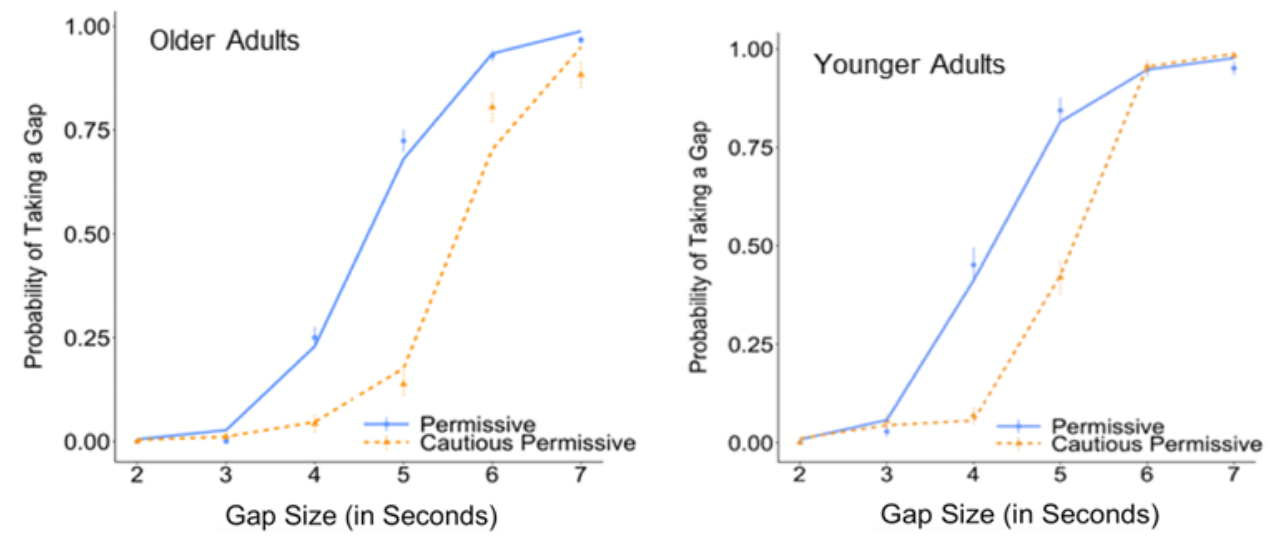

Figure 5: Logistic regression curves modeling the probability of taking a gap as a function of age, condition, and gap size for older adults (left) and younger adults (right).

Table 6: Means and standard deviations (in parentheses) of movement timing measures (in seconds) for each condition.

\begin{tabular}{lccr}
\hline Condition & Timing of entry & Road crossing time & \multicolumn{1}{c}{ Time to spare } \\
\hline Permissive & $.72(.21)$ & $2.14(.26)$ & $3.05(.95)$ \\
Cautious permissive & $.78(.21)$ & $2.19(.32)$ & $3.30(.84)$ \\
\hline
\end{tabular}

Table 7: The number of trials that older and younger adults complied or did not comply with the cautious permissive alerts.

\begin{tabular}{lcccc}
\hline Cautious Status & Older: did not cross & Older: crossed & Younger: did not cross & Younger: crossed \\
\hline Cautious Alert Off & 349 & 17 & 440 & 71 \\
Cautious Alert On & 36 & 193 & 10 & 329 \\
\hline
\end{tabular}

people were more likely to cross when the gaps were alerted than in the original permissive alerts condition.

\subsection{Qualitative Results}

8.3.1 Categorical Responses. Participants reported whether they noticed the visual, auditory, or vibratory element of the cautious permissive alerts, and which of those elements was the most prominent. There were no statistically significant differences between the conditions when looking at each age group (see Figure 6 for percentages).

8.3.2 Open-Ended Responses. Both older adult (OCPe1-OCPe10) and younger adult (YCPe1-YCPe20) participants had more positive comments about the cautious permissive alerts $(n=72)$ than negative $(n=40)$. We again found that $3 / 10$ older adults and $3 / 20$ younger adults mentioned that the alerts resulted in them becoming less attentive to the road. Also, while themes of helpfulness and trust came up both positively and negatively, a third theme was prominent: safety. In particular, $6 / 10$ older adults mentioned that they appreciated the safety of the cautious permissive alerts, while that was only true for $5 / 20$ younger adults. In contrast, only one older adult mentioned that the cautious permissive alerts were too safe, while this was true for $9 / 20$ younger adults. OCPe10 said, “. . and then several times the alert did not come after the second car and I just went anyway." YCPe2 said "a couple times I felt like I could've crossed when it didn't say it was safe to cross." 


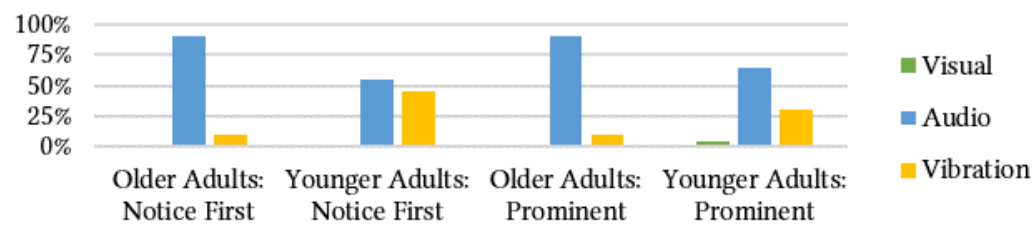

Figure 6: Percentage of participants who reported whether they noticed visual (left, green), audio (middle, blue), or vibration (right, yellow) first or thought they were most prominent for the cautious permissive alerts.

\section{DISCUSSION}

This paper examined two approaches to the design of vehicle-topedestrian (V2P) technology to assist pedestrians in crossing traffic: permissive alerts, that signal when it is safe to cross, and prohibitive warnings, that signal when it is not safe to cross. We found that both older and younger adults were highly likely to heed permissive alerts, even when this meant taking smaller gaps than they would typically without alerts (i.e., controls). A follow-up condition showed that we could easily shift participants' road-crossing behavior toward greater caution when we only alerted them to cross very large gaps. That said, both older and younger adults were more likely to cross when not alerted to do so (or 'errors of commission') in the cautious permissive condition than in the ability-based permissive condition. We also found that participants reported the prohibitive warnings as annoying, unlike the permissive alerts. These findings give insights into V2P design and about how both younger and older adults respond to smartphone alerts and warnings. We conclude with a discussion of the similarities and differences between older and younger adults, design considerations for eHMI and V2P technologies, limitations of the research, and opportunities for future work.

\subsection{Age-Related Similarities and Differences}

The younger and older adult participants showed very similar patterns of behavior in their responses to the permissive alerts and prohibitive warnings. Both age groups complied with the permissive alerts and showed similar shifts in their gap thresholds in the cautious permissive condition relative to the original permissive condition. However, there were some nuances to the quantitative and qualitative findings for older and younger adults. First, older adults selected larger gaps than younger adults across all conditions. Second, younger adults were divided as to whether they first noticed the sound or the vibration, while most older adults first noticed the sound. Finally, older adults appreciated the safety of the cautious permissive alerts, whereas younger adults felt like they were too safe. We conclude that having multiple modalities is the best approach, which matches Mahadevan et al.'s findings on autonomous vehicle-pedestrian communications [23].

\subsection{Design Considerations for V2P Communications}

Participants were highly likely to follow ability-based permissive alerts, regardless of whether they were alerted to take gaps that differed from what they would typically take on their own. (Note that participants safely crossed the roadway with ample time to spare even when they followed these permissive alerts.) Further, permissive alert compliance was true despite age-related differences in alerts given (and gap selection). One potential reason the abilitybased permissive alerts had more of an influence than prohibitive warnings is because participants found the warnings annoying. Another reason is that people respond better to positively framed messaging, as seen in Choe et al.'s finding that positively framed step count progress increases people's self-efficacy in completing a step count goal [8]. Also, adults and children prefer eHMI signals that tell them what to do (e.g., "walk") than those that tell them what not to do (e.g., "stop") [10]. These notions are confirmed through self-reported quotes that ability-based permissive participants sometimes took smaller gaps than they would have otherwise. More alarmingly, some older and younger participants using alerts and warnings self-reported that they were less attentive to the virtual road. This is consistent with previous research showing that participants who received alerts and warnings while texting and crossing roads spent much less time looking at the traffic than participants who did not receive alerts and warnings [35]. When participants place enough trust into V2P communications, they are at risk of becoming over reliant on the technology. Issues of overreliance on navigation-based technologies can have dire consequences, including people following GPS driving directions into a lake [19] or men falling off a cliff while playing Pokémon Go [11]. V2P communications must communicate safety messaging to pedestrians that these tools are meant to augment their abilities, not replace them. App developers should also consider how such systems might reduce pedestrians' situational awareness.

\subsection{Informing the Timing of Alerts and Warnings}

Our results underscore the difficulty of providing useful prohibitive warnings to pedestrians. Prior research demonstrated that warnings were ineffective when sent after participants had initiated a crossing movement [36]. Here, we attempted to warn participants that crossing opportunities were risky before they began to cross. We timed the verbal warnings so that the first spoken warning of "Do not Cross" ended just before the dangerous gap opened and repeated every two seconds up until a safe gap arrived at the intersection. Because participants were likely focusing on the upcoming safe gap while hearing the phrase "Do not cross" repeated, there is a possibility they thought the warning applied to the upcoming safe gap instead of gap immediately in front of them. Due to the difficulty of linking the prohibitive warnings to the correct gap, we 
recommend that designers avoid a "Do not Cross" approach; not only were these warnings reported as annoying, but they could also confuse users and place them at greater risk for collisions.

We also timed the ability-based permissive alerts such that the verbal indication of "Safe to Cross" was complete just before the gap opened. However, when the alert occurred, there was still a car in front of the person, which could have caused confusion. Because people at times had difficulty linking alerts to the correct gap, we suggest visually designing alerts directly on the roadway. For example, integrating V2P technology with pedestrian-worn AR glasses would allow a person to pay full attention to traffic and view highlighted gaps that reflect crossability. By visually designating crossability, people would not have to map auditory messages to visually perceived gaps. While this is a stretch with current AR technology, it will likely be feasible in the future given the announcement of Facebook's AR glasses [15], plus Apple's rumored future release [24].

\subsection{Limitations and Future Work}

While we were careful in the design of the app and task, there are limitations to this study. First, our study population came from an active and highly educated community so our findings may not generalize to all older and younger adults. Although we reasoned that a one-size-fits-all approach to alerts and warnings may not work due to older adults' slower walking speeds, we did not find large differences in crossing times between our older and younger participants. This is likely because the large-screen virtual environment constrained the crossing distance, and our older adults were active and healthy. While we established findings for non-mobilityimpaired older and younger adults, future work should involve adults who use mobility aids. Second, the cars all drove at the same speed and came from one direction, which made the virtual roadcrossing task simpler than real-world road crossing. Future work could continue to study how people respond to V2P communication in more realistic and complex street-crossing settings, including the roads with higher and varied car speeds and where cars come from multiple directions. We delivered the information in multiple modalities to maximize perceptibility in the face of the spatialized traffic sounds, but real-world conditions may further challenge perceptibility of alerts and warnings from a handheld device [21,38]. Third, in the real world, V2P technology will not uniformly deploy to all cars at once; there will exist times in which some cars have this capability and others do not. This may limit the usefulness and safety of communicating alerts and warnings to pedestrian smartphones.

Finally, as with all simulation studies, there is a question about ecological validity. Mullen et al. [25] surveyed the research on the validity of driving simulators and concluded that "simulator driving behavior approximates (relative validity), but does not exactly replicate (absolute validity), on-road driving behavior." Likewise in their review of pedestrian behavior in virtual environments, Schneider and Benger [38] concluded that "results should be cautiously understood in terms of relative, not absolute validity..." Based on this large body of research on driving and pedestrian simulation, we believe that people are likely to heed permissive alerts in the real world. However, compliance with permissive alerts may be reduced given the life and death consequences of making an error.

\section{CONCLUSION}

The frequency of V2P and eHMI technologies are increasing in both research and practice. Thus, it is important to understand how alerts and warnings can influence the street-crossing behavior of pedestrians. We designed two smartphone systems, permissive alerts and prohibitive warnings, to understand their effect on younger and older pedestrians. We conducted a between-subjects study to compare permissive alerts, prohibitive warnings, and a control group and found that participants took smaller gaps with the permissive alerts than they would typically take on their own. We followed with a "cautious" permissive condition, where we alerted only large gaps and found that participants largely complied with the alerts and took larger gaps than in the original permissive condition. Our findings can inform the design of future V2P communications technology.

\section{ACKNOWLEDGMENTS}

This work was supported in part by Safety Research Using Simulation University Transportation Center (SAFER-SIM) 69A3551747131 and National Science Foundation grants IIS-1849822 and CNS1305131.

\section{REFERENCES}

[1] Sander Ackermans, Debargha Dey, Peter Ruijten, Raymond H. Cuijpers, and Bastian Pfleging. 2020. The Effects of Explicit Intention Communication, Conspicuous Sensors, and Pedestrian Attitude in Interactions with Automated Vehicles. In Proceedings of the $2020 \mathrm{CHI}$ Conference on Human Factors in Computing Systems (CHI '20). Association for Computing Machinery, Honolulu, HI, USA. 1-14. https://doi.org/10.1145/3313831.3376197

[2] José Javier Anaya, Pierre Merdrignac, Oyunchimeg Shagdar, Fawzi Nashashibi, and José E. Naranjo. 2014. Vehicle to Pedestrian Communications for Protection of Vulnerable Road Users. In 2014 IEEE Intelligent Vehicles Symposium. Dearborn, Michigan, United States. 1-6. https://doi.org/10.1109/ivs.2014.6856553

[3] G. Oscar Anderson. 2018. Getting Connected: Older Americans Embrace Technology to Enhance Their Lives. AARP. https://doi.org/10.26419/res.00210.001

[4] Monica Anderson and Andrew Perrin. 2017. Technology use among seniors. Pew Research Center: Internet, Science \& Tech. Retrieved August 15, 2019 from https://www.pewinternet.org/2017/05/17/technology-use-among-seniors/

[5] Farah Arab, Sabrina Panëels, Margarita Anastassova, Stéphanie Coeugnet, Fanny Le Morellec, Aurélie Dommes, and Aline Chevalier. 2015. Haptic patterns and older adults: To repeat or not to repeat? In 2015 IEEE World Haptics Conference (WHC). 248-253. https://doi.org/10.1109/WHC.2015.7177721

[6] BMW Group. New centre of excellence for autonomous driving. BMW officially opens its autonomous driving campus in Unterschleißheim near Munich. Retrieved August 3, 2020 from https://www.press.bmwgroup.com/global/article/ detail/T0280021EN/new-centre- of-excellence-for-autonomous-driving-bmwofficially-opens-its-autonomous-driving-campus-in- unterschleissheim-nearmunich?language $=$ en

[7] P. Burnard. 1991. A method of analysing interview transcripts in qualitative research. Nurse Educ Today 11, 6 (December 1991), 461-466. https://doi.org/10. 1016/0260-6917(91)90009-y

[8] Eun Kyoung Choe, Bongshin Lee, Sean Munson, Wanda Pratt, and Julie A. Kientz. 2013. Persuasive Performance Feedback: The Effect of Framing on Self-Efficacy. AMIA Annu Symp Proc 2013, (November 2013), 825-833.

[9] Carolina Cruz-Neira, Daniel J. Sandin, and Thomas A. DeFanti. 1993. Surroundscreen projection-based virtual reality: the design and implementation of the CAVE. In Proceedings of the 20th annual conference on Computer graphics and interactive techniques - SIGGRAPH '93. ACM Press, Not Known. 135-142. https://doi.org/10.1145/166117.166134

[10] Shuchisnigdha Deb, Daniel W. Carruth, Muztaba Fuad, Laura M. Stanley, and Darren Frey. 2020. Comparison of Child and Adult Pedestrian Perspectives of External Features on Autonomous Vehicles Using Virtual Reality Experiment. In Advances in Human Factors of Transportation (Advances in Intelligent Systems 
and Computing). Springer International Publishing, Cham. 145-156. https://doi. org/10.1007/978-3-030-20503-4_13

[11] Janissa Delzo. Men fall from cliff playing Pokémon Go - CNN. CNN Health Retrieved September 4, 2020 from https://www.cnn.com/2016/07/15/health/ pokemon-go-players-fall-down-cliff/index.html

[12] Aurélie Dommes and Viola Cavallo. 2011. The role of perceptual, cognitive, and motor abilities in street-crossing decisions of young and older pedestrians. Ophthalmic and Physiological Optics 31, 3 (2011), 292-301. https://doi.org/10. 1111/j.1475-1313.2011.00835.x

[13] Aurélie Dommes, Viola Cavallo, and Jennifer Oxley. 2013. Functional declines as predictors of risky street-crossing decisions in older pedestrians. Accident Analysis \& Prevention 59, (October 2013), 135-143. https://doi.org/10.1016/j.aap. 2013.05.017

[14] Stefanie M. Faas, Andrea C. Kao, and Martin Baumann. 2020. A Longitudinal Video Study on Communicating Status and Intent for Self-Driving Vehicle-Pedestrian Interaction. In Proceedings of the $2020 \mathrm{CHI}$ Conference on Human Factors in Computing Systems (CHI '20). Association for Computing Machinery, New York, NY, USA. 1-14. https://doi.org/10.1145/3313831.3376484

[15] Facebook. 2020. Facebook Connect: The Road to AR Glasses. Facebook Technology. Retrieved December 21, 2020 from https://tech.fb.com/facebook-connectthe-road-to-ar-glasses/

[16] Yasmin Felberbaum, Joel Lanir, and Patrice L. (Tamar) Weiss. 2018. Challenges and Requirements for Technology to Support Mobility of Older Adults. In Extended Abstracts of the $2018 \mathrm{CHI}$ Conference on Human Factors in Computing Systems (CHI EA '18). ACM, New York, NY, USA. LBW545:1-LBW545:6. https://doi.org/ $10.1145 / 3170427.3188637$

[17] Ahmed Hussein, Fernando García, Jose M. Armingol, and Cristina OlaverriMonreal. 2016. P2V and V2P communication for Pedestrian warning on the basis of Autonomous Vehicles. In 2016 IEEE 19th International Conference on Intelligent Transportation Systems (ITSC). 2034-2039. https://doi.org/10.1109/ ITSC.2016.7795885

[18] American Honda Motor Co Inc. Honda Demonstrates Advanced Vehicleto-Pedestrian and Vehicle-to-Motorcycle Safety Technologies. Retrieved September 14, 2020 from https://www.prnewswire.com/news-releases/hondademonstrates-advanced-vehicle-to-pedestrian-and-vehicle-to-motorcyclesafety-technologies-221495031.html

[19] Madison Malone Kircher. 2018. Yet Another Person Listens to GPS App and Drives Car Into Lake. Intelligencer. Retrieved September 4, 2020 from https://nymag.com/intelligencer/2018/01/waze-app-directs-driverto-drive-car-into-lake-champlain.html

[20] Jason Laberge, Janet Creaser, Michael Rakauskas, and Nicholas Ward. 2006. De sign of an Intersection Decision Support (IDS) interface to reduce crashes at rural stop-controlled intersections. Transportation Research Part C: Emerging Technologies 14, (February 2006), 39-56. https://doi.org/10.1016/j.trc.2006.03.001

[21] Grégoire S. Larue, Christian Wullems, Michelle Sheldrake, and Andry Rakotonirainy. 2018. Validation of a Driving Simulator Study on Driver Behavior at Passive Rail Level Crossings. Hum Factors 60, 6 (September 2018), 743-754. https://doi.org/10.1177/0018720818783507

[22] Phil LeBeau. 2019. Pedestrian deaths hit 28-year high, and big vehicles and smartphones are to blame. CNBC. Retrieved August 15, 2019 from https://www.cnbc.com/2019/02/28/pedestrian-deaths-hit-a-28-year-highand-big-vehicles-and-smartphones-are-to-blame.html

[23] Karthik Mahadevan, Sowmya Somanath, and Ehud Sharlin. 2018. Communicating Awareness and Intent in Autonomous Vehicle-Pedestrian Interaction. 1-12. https: //doi.org/10.1145/3173574.3174003

[24] Carrie Mihalcik. Apple Glass: Major leak may spill details on long-rumored AR glasses. CNET. Retrieved December 21, 2020 from https://www.cnet.com/news/ apple-glass-major-leak-may-spill-details-on-long-rumored-ar-glasses/

[25] Nadia Mullen, Judith Charlton, Anna Devlin, and Michel Bedard. 2011. Simulator validity: Behaviours observed on the simulator and on the road. Handbook of Driving Simulation for Engineering, Medicine and Psychology (2011), 1-18.

[26] National Center for Statistics and Analysis. 2020. Pedestrians: 2018 data (Traffic Safety Facts. Report No. DOT HS 812 850). National Highway Traffic Safety Administration.

[27] National Highway Traffic Safety Administration. 2017. Federal Motor Vehicle Safety Standards; V2V Communications. Federal Register 82, 8 (January 2017),
3854-4019.

[28] Janick Naveteur, J. Delzenne, Pascal Sockeel, Éric Watelain, and Marie Agnès Dupuy. 2013. Crosswalk time estimation and time perception: An experimental study among older female pedestrians. Accident Analysis \& Prevention 60, (November 2013), 42-49. https://doi.org/10.1016/j.aap.2013.08.014

[29] NYC Connected Vehicle Project. Project Scope. Retrieved September 14, 2020 from https://www.cvp.nyc/project-scope

[30] Steve O'Hern, Jennifer Oxley, and David Logan. 2015. Older Adults at Increased Risk as Pedestrians in Victoria, Australia: An Examination of Crash Characteristics and Injury Outcomes. Traffic Inj Prev 16 Suppl 2, (2015), S161-167. https://doi.org/10.1080/15389588.2015.1061662

[31] Elizabeth E. O'Neal, Yuanyuan Jiang, Lucas J. Franzen, Pooya Rahimian, Junghum Paul Yon, Joseph K. Kearney, and Jodie M. Plumert. 2018. Changes in perceptionaction tuning over long time scales: How children and adults perceive and act on dynamic affordances when crossing roads. Journal of Experimental Psychology: Human Perception and Performance 44, 1 (2018), 18-26. https://doi.org/10.1037/ xhp0000378

[32] Jennifer A. Oxley, Elfriede Ihsen, Brian N. Fildes, Judith L. Charlton, and Ross H. Day. 2005. Crossing roads safely: An experimental study of age differences in gap selection by pedestrians. Accident Analysis \& Prevention 37, 5 (September 2005), 962-971. https://doi.org/10.1016/j.aap.2005.04.017

[33] PAR, Inc. Mini-Mental State Examination Second Edition | MMSE-2. Retrieved September 10, 2019 from https://www.parinc.com/Products/Pkey/238

[34] Qualtrics. Qualtrics XM - Experience Management Software. Qualtrics. Retrieved December 21, 2020 from https://www.qualtrics.com/

[35] Pooya Rahimian, Elizabeth O'Neal, Junghum Paul Yon, Luke Franzen, Yuanyuan Jiang, Jodie Plumert, and Joseph K. Kearney. 2016. Using a virtual environment to study the impact of sending traffic alerts to texting pedestrians. In IEEE Virtual Reality (VR). 141-149. https://doi.org/10.1109/VR.2016.7504697

[36] Pooya Rahimian, Elizabeth O'Neal, Shiwen Zhou, Jodie Plumert, and Joseph K. Kearney. 2018. Harnessing Vehicle-to-Pedestrian (V2P) Communication Technology: Sending Traffic Warnings to Texting Pedestrians. Human Factors: The Journal of the Human Factors and Ergonomics Society 60, (June 2018), 001872081878136. https://doi.org/10.1177/0018720818781365

[37] Linda Rothman, Andrew William Howard, Andi Camden, and Colin Macarthur. 2012. Pedestrian crossing location influences injury severity in urban areas. Inj. Prev. 18, 6 (December 2012), 365-370. https://doi.org/10.1136/injuryprev-2011040246

[38] Sonja Schneider and Klaus Bengler. 2020. Virtually the same? Analysing pedestrian behaviour by means of virtual reality. Transportation Research Part F: Traffic Psychology and Behaviour 68, (January 2020), 231-256. https://doi.org/10. 1016/j.trf.2019.11.005

[39] Steven E. Shladover. 2018. Connected and automated vehicle systems: Introduction and overview. Journal of Intelligent Transportation Systems 22, 3 (May 2018), 190-200. https://doi.org/10.1080/15472450.2017.1336053

[40] Anne Shumway-Cook, Aftab Patla, Anita Stewart, Luigi Ferrucci, Marcia A. Ciol, and Jack M. Guralnik. 2003. Environmental Components of Mobility Disability in Community-Living Older Persons. Journal of the American Geriatrics Society 51, 3 (2003), 393-398. https://doi.org/10.1046/j.1532-5415.2003.51114.x

[41] United States Department of Transportation. Intelligent Transportation Systems - Connected Vehicle: Vehicle-to-Pedestrian (V2P) Communications. Retrieved January 8, 2021 from https://www.its.dot.gov/factsheets/cv_v2pcomms.htm

[42] US Department of Transportation. USDOT Automated Vehicles Activities. Retrieved August 3, 2020 from https://www.transportation.gov/AV

[43] Kyle Wiggers. 2020. 77 autonomous vehicles drove over 500,000 miles across Beijing in 2019. VentureBeat. Retrieved August 3, 2020 from https://venturebeat.com/2020/03/02/77-autonomous-vehicles-drove-over500000-miles-across-beijing-in-2019/

[44] World Health Organization. WHO | Global status report on road safety 2018. WHO. Retrieved August 15, 2019 from http://www.who.int/violence_injury_ prevention/road_safety_status/2018/en/

[45] G. A. Zito, D. Cazzoli, L. Scheffler, M. Jäger, R. M. Müri, U. P. Mosimann, T. Nyffeler, F. W. Mast, and T. Nef. 2015. Street crossing behavior in younger and older pedestrians: an eye- and head-tracking study. BMC Geriatrics 15, 1 (December 2015), 176. https://doi.org/10.1186/s12877-015-0175-0 